\title{
Multiple Crossover per Couple with Selection of the Two Best Offspring: An Experimental Study with the BLX-a Crossover Operator for Real-Coded Genetic Algorithms
}

\author{
F. Herrera ${ }^{1}$, M. Lozano ${ }^{1}$, E. Pérez ${ }^{2}$, A.M. Sánchez ${ }^{3}$, and P. Villar ${ }^{3}$ \\ ${ }^{1}$ Dpto. de Ciencias de la Computación e Inteligencia Artificial, Escuela Técnica Superior de \\ Ingeniería Informática, Universidad de Granada \\ \{herrera, lozano\} @decsai.ugr.es \\ 2 Grupo de Ingeniería Industrial, ETS de Ingeniería Industrial, Universidad de Valladolid \\ elena@eis.uva.es \\ 3 Dpto. de Informática, Escuela Superior de Ingeniería Informática, Universidad de Vigo \\ \{amlopez, pvillar\}@uvigo.es
}

\begin{abstract}
In this paper, we propose a technique for the application of the crossover operator that generates multiple descendants from two parents and selects the two best offspring to replace the parents in the new population. In order to study the proposal, we present different instances based on the BLX- $\alpha$ crossover operator for real-coded genetic algorithms. In particular, we investigate the influence of the number of generated descendants in this operator, the number of evaluations, and the value for the parameter $\alpha$. Analyzing the experimentation that we have carried out, we can observe that it is possible, with multiple descendants, to achieve a suitable balance between the explorative properties associated with BLX- $\alpha$ and the high selective pressure associated to the selection of the two best descendants.
\end{abstract}

\section{Introduction}

An important objective in the development of the genetic operators is to keep a suitable balance between exploration and exploitation, and therefore, to obtain good solutions in the searching process. In this paper, we study real-coded genetic algorithms (RCGAs) ([10]) paying special attention to the way the crossover operator is applied, in order to achieve the balance between exploration and exploitation.

There are different proposals of crossover operators and, particularly, there are crossover operators that generate three ([15]) and four descendants ([8,9]) from two parents and then, they select the two best descendants to replace the parents in the population. In these works, the operators with multiple descendants, more than two descendants 
from two parents, present a better behavior than the operators with only two descendants, and achieve a good balance between exploration and exploitation.

According to these ideas, in this paper, we present a proposal for the generation of multiple descendants using the BLX- $\alpha$ crossover operator ([4]) for RCGAs, which operates over two parents, selecting the two best ones to replace the parents in the new population.

The BLX- $\alpha$ operator has associated a high exploration, which induces a high diversity among the descendants. Therefore, this operator allows big differences among the descendants and among them and their parents. On the other hand, the possibility of generating multiple descendants and afterwards selecting the two best ones introduces a high selective pressure.

Due to these reasons, we present the proposal of multiples descendants for the BLX- $\alpha$ crossover operator, introducing a higher selective pressure on this operator with the selection of the two best descendants. With this combination, we can achieve an effective balance between exploitation and exploration and therefore, better solutions may be reached.

We have set up the paper as follows. In Section 2, we describe the BLX- $\alpha$ crossover operator and analyze its behavior when it generates two descendants for different values of $\alpha$ and different population sizes. In Section 3, we present the proposal of multiple descendants and selection of the two best. In Section 4, the experimental study and the analysis of the operator according to the number of generated descendants and to the value of the $\alpha$ parameter is showed. In Sections 5, we study the importance of selecting the two best descendents. In Section 6, we investigate the performance of the model proposed for different numbers of evaluations. Finally, some conclusions are presented in Section 7.

\section{The BLX-a Crossover Operator for RCGAs}

Let us assume that $\mathrm{C}_{1}=\left(\mathrm{c}_{1}{ }^{1}, \ldots, \mathrm{c}_{\mathrm{n}}{ }^{1}\right)$ and $\mathrm{C}_{2}=\left(\mathrm{c}_{1}{ }^{2}, \ldots, \mathrm{c}_{\mathrm{n}}{ }^{2}\right)$ are two real-coded chromosomes to be crossed, then BLX- $\alpha$ generates two offspring, $\mathrm{H}_{\mathrm{k}}=\left(\mathrm{h}_{1}{ }^{\mathrm{k}}, \ldots, \mathrm{h}_{\mathrm{i}}{ }^{\mathrm{k}}, \ldots, \mathrm{h}_{\mathrm{n}}{ }^{\mathrm{k}}\right), \mathrm{k}$ $=1,2$, where $\mathrm{h}_{\mathrm{i}}{ }^{\mathrm{k}}$ is a randomly (uniformly) chosen number from the interval $\left[\mathrm{c}_{\min }-\mathrm{I} \alpha\right.$, $\left.\mathrm{c}_{\max }+\mathrm{I} \alpha\right]$, where $\mathrm{c}_{\max }=\max \left\{\mathrm{c}_{\mathrm{i}}{ }^{1}, \mathrm{c}_{\mathrm{i}}{ }^{2}\right\}, \mathrm{c}_{\min }=\min \left\{\mathrm{c}_{\mathrm{i}}{ }^{1}, \mathrm{c}_{\mathrm{i}}{ }^{2}\right\}$, and $\mathrm{I}=\mathrm{c}_{\max }-\mathrm{c}_{\min }$. Figure 1 shows the effect of this operator.

It is important to emphasize that this crossover operator is based on the random generation of genes from the associated neighborhood of the genes in the parents. With this technique for the generation of genes, offspring are generated that can defer among them and also among them and their parents. In this sense, this operator presents a high exploration in the generation of the descendants. 


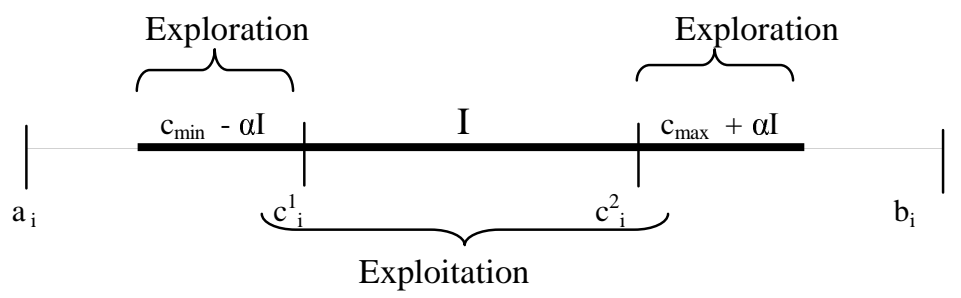

Fig. 1. Interval of random generation for a gene with BLX- $\alpha$

We study empirically the behavior of BLX- $\alpha$ with different values for $\alpha(0.0,0.1,0.3$, 0.5 , and 0.7$)$ and for the population size $(N=61$ and $N=100)$. In these experiments, BLX- $\alpha$ generates two descendants, which replace the parents in the population.

We have considered thirteen frequently used test functions: Sphere model, Schwefel's problem, Generalized Rastrigin's function, Griewangk's function, Expansion of F10, Generalized Rosenbrock's function, system of linear equations, frequency modulation sounds parameter identification problem, polynomial fitting problem, Ackley's function, Bohachevsky's function, Watson's function, and Colville's function. The formulation of these problems may be found in [11,13]. These functions defer with respect to some characteristics like continuity, modality or dimensions. In this way, the interval of possible situations is wide enough to reach a good level of robustness in experimentation. The dimension of the search space is 25 .

A generational GA model is assumed that applies the non-uniform mutation operator ([12]). The selection probability calculation follows linear ranking ([1]) and the sampling algorithm is the stochastic universal sampling ([2]). The elitist strategy is considered as well. We executed all the algorithms 30 times, each one with a maximum of 100000 evaluations. The crossover probability is 0.6 and the probability of updating a chromosome by mutation is 0.1 .

Tables 1 and 2 present a summary of the results with 61 and 100 chromosomes, respectively. We have compared, using t-test (at 0.05 level of significance), the best result achieved by the operator, according to the value of $\alpha$, with the rest of results using the other $\alpha$ values for each function. These tables show the percentages over the total of functions in which the operator has obtained the best results. Each column has the following information:

- BA / BT: Best Average / Best Test. Percentage of evaluation functions in which the crossover operator has obtained the best average value and continues being the best one after the application of the t-test.

- BA / ST: Best Average / Similar Test. Percentage of evaluation functions in which the crossover operator has obtained the best average value and, at least there is another crossover operator that does not present significant differences after the application of the t-test. 
- TB: Percentage of evaluation functions in which the crossover operator presents better results without considering the information that we have obtained by the application of the t-test. This percentage is the result of the addition of the two previous columns.

- ST / NBA: Percentage of evaluation functions in which the crossover operator shows a similar behavior to the best crossover operator after the application of the $\mathrm{t}$ test, although it does not obtain the best average value.

- T B / S: Percentage of evaluation functions in which the crossover operator shows the best behavior or similar to the best. This percentage is the result of the addition of the two previous columns.

According to these results, we can observe that BLX- $\alpha$ achieves the best result when $\alpha$ is 0.3 , independently of the population size. This value allows some descendants in the exploration intervals to be generated, introducing diversity in the population. We have compared the results obtained with each parameter, for the different population sizes, and we have observed that the results are better with 61 chromosomes.

Table 1. Results obtained by BLX- $\alpha$ with different values of $\alpha$ and $N=61$

\begin{tabular}{|c|c|c|c|c|c|}
\hline Crossover & BA/BT & BA/ST & TB & ST/NBA & T B/S \\
\hline BLX-0.0 & $0 \%$ & $7.69 \%$ & $\mathbf{7 . 6 9 \%}$ & $38.45 \%$ & $\mathbf{4 6 . 1 4 \%}$ \\
\hline BLX-0.1 & $0 \%$ & $0 \%$ & $\mathbf{0 \%}$ & $53.83 \%$ & $\mathbf{5 3 . 8 3 \%}$ \\
\hline BLX-0.3 & $38.45 \%$ & $53.83 \%$ & $\mathbf{9 2 . 2 8 \%}$ & $0 \%$ & $\mathbf{9 2 . 2 8 \%}$ \\
\hline BLX-0.5 & $0 \%$ & $0 \%$ & $\mathbf{0 \%}$ & $38.45 \%$ & $\mathbf{3 8 . 4 5 \%}$ \\
\hline BLX-0.7 & $0 \%$ & $0 \%$ & $\mathbf{0 \%}$ & $7.69 \%$ & $\mathbf{7 . 6 9 \%}$ \\
\hline
\end{tabular}

Table 2. Results obtained by BLX- $\alpha$ with different values of $\alpha$ and $N=100$

\begin{tabular}{|c|c|c|c|c|c|}
\hline Crossover & BA/BT & BA/ST & TB & ST/NBA & T B/S \\
\hline BLX-0.0 & $7.69 \%$ & $0 \%$ & $\mathbf{7 . 6 9 \%}$ & $30.76 \%$ & $\mathbf{3 8 . 4 5 \%}$ \\
\hline BLX-0.1 & $0 \%$ & $0 \%$ & $\mathbf{0 \%}$ & $53.83 \%$ & $\mathbf{5 3 . 8 3 \%}$ \\
\hline BLX-0.3 & $30.76 \%$ & $53.83 \%$ & $\mathbf{8 4 . 5 9 \%}$ & $15.38 \%$ & $\mathbf{1 0 0 \%}$ \\
\hline BLX-0.5 & $0 \%$ & $7.69 \%$ & $\mathbf{7 . 6 9 \%}$ & $7.69 \%$ & $\mathbf{1 5 . 3 8 \%}$ \\
\hline BLX-0.7 & $0 \%$ & $7.69 \%$ & $\mathbf{7 . 6 9 \%}$ & $7.69 \%$ & $\mathbf{1 5 . 3 8 \%}$ \\
\hline
\end{tabular}

\section{Generation of Multiple Descendants and Selection of the Two Best}

In this section, we propose to increase the number of descendants generated by the BLX- $\alpha$ operator, leading to the mechanism of generation of multiple descendants with the selection of the two best to replace the parents in the new population. It will be denoted as GMD+STB.

The GMD+STB is possible by means of an unique operator or by the hybridization of different crossover operators, using a different operator for each descendant or group 
of descendants. In this paper, we will use an unique operator, BLX- $\alpha$, to obtain all the descendants, and we will consider the possible extensions for future works.

The mechanism proposed generates 4, 6, $8,16,32,64$, and 128 descendants for each pair of parents, and selects the two best (operator 2-2-2, 2-4-2, 2-6-2, 2-8-2, 2-16-2, 2$32-2,2-64-2$, and 2-128-2, respectively) to be included in the new population. Figure 2 shows graphically the working scheme of this mechanism.

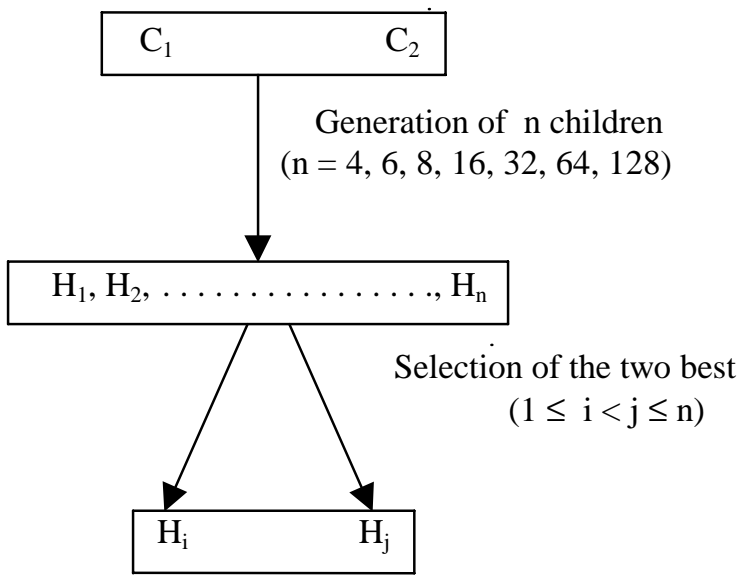

Fig. 2. Scheme for the mechanism of GMD+STB

The generation of multiple descendants from two parents has been considered in different proposals. In [5,6], multiple descendants are generated from two parents using different crossover operators, introducing all the descendants in the new population (MCPC model). In [7], a single offspring from the multiple crossovers (MCPC and MCMP) is selected and inserted in the next population. In [14], the generation of multiple descendants is proposed, selecting the two chromosomes for the next population from the named family, the parents and their children (MGG model). In [3], a generalization of this model is presented. In this case, one of the chromosomes is the best one of the population and another is selected by a classical sampling mechanism (or various ones in the case of multiple parents) and, taking them as point of departure, multiple descendants are generated; finally, the two best chromosomes of the family are selected (G3 model).

The proposal defers from these models because it has a selection of descendants and the parents do not participate in the selection process.

\section{The BLX-a Crossover Operator with GMD+STB}

In this section, we study empirically the effects of the GMD+STB, when the number of descendant is incremented, for the BLX- $\alpha$ operator with $\alpha=0.5,0.3$, and 0.1 . These 
values allow to use intervals of exploration and produce different levels of diversity. The population size is 61 . Tables 3, 4, and 5, and Figure 4, present a summary of the results for the parameters $0.5,0.3$, and 0.1 respectively. These tables show the percentages over the total of functions in which the operator, according to the number of descendants, has obtained the best results.

Table 3. Results obtained by BLX-0.5 with GMD+STB

\begin{tabular}{|c|c|c|c|c|c|}
\hline BLX 0.5 & BA/BT & BA/ST & TB & ST/NBA & T B/S \\
\hline $\mathbf{2 - 2 - 2}$ & $0 \%$ & $0 \%$ & $\mathbf{0 \%}$ & $23.07 \%$ & $\mathbf{2 3 . 0 7 \%}$ \\
\hline $\mathbf{2 - 4 - 2}$ & $0 \%$ & $15.38 \%$ & $\mathbf{1 5 . 3 8 \%}$ & $30.76 \%$ & $\mathbf{4 6 . 1 4 \%}$ \\
\hline $\mathbf{2 - 6 - 2}$ & $0 \%$ & $7.69 \%$ & $\mathbf{7 . 6 9 \%}$ & $46.14 \%$ & $\mathbf{5 3 . 8 3 \%}$ \\
\hline $\mathbf{2 - 8 - 2}$ & $23.07 \%$ & $23.07 \%$ & $\mathbf{4 6 . 1 4 \%}$ & $30.76 \%$ & $\mathbf{7 6 . 9 0 \%}$ \\
\hline $\mathbf{2 - 1 6 - 2}$ & $7.69 \%$ & $23.07 \%$ & $\mathbf{3 0 . 7 6 \%}$ & $30.76 \%$ & $\mathbf{6 1 . 5 2 \%}$ \\
\hline $\mathbf{2 - 3 2 - 2}$ & $7.69 \%$ & $15.38 \%$ & $\mathbf{2 3 . 0 7 \%}$ & $23.07 \%$ & $\mathbf{4 6 . 1 4 \%}$ \\
\hline $\mathbf{2 - 6 4 - 2}$ & $0 \%$ & $0 \%$ & $\mathbf{0 \%}$ & $30.76 \%$ & $\mathbf{3 0 . 7 6 \%}$ \\
\hline $\mathbf{2 - 1 2 8 - 2}$ & $0 \%$ & $0 \%$ & $\mathbf{0 \%}$ & $30.76 \%$ & $\mathbf{3 0 . 7 6 \%}$ \\
\hline
\end{tabular}

Table 4. Results obtained by BLX-0.3 with GMD+STB

\begin{tabular}{|c|c|c|c|c|c|}
\hline BLX 0.3 & BA/BT & BA/ST & TB & ST/NBA & T B/S \\
\hline $\mathbf{2 - 2 - 2}$ & $0 \%$ & $0 \%$ & $\mathbf{0 \%}$ & $30.76 \%$ & $\mathbf{3 0 . 7 6 \%}$ \\
\hline $\mathbf{2 - 4 - 2}$ & $0 \%$ & $15.38 \%$ & $\mathbf{1 5 . 3 8 \%}$ & $23.07 \%$ & $\mathbf{3 8 . 4 5 \%}$ \\
\hline $\mathbf{2 - 6 - 2}$ & $0 \%$ & $7.69 \%$ & $\mathbf{7 . 6 9 \%}$ & $30.76 \%$ & $\mathbf{3 8 . 4 5 \%}$ \\
\hline $\mathbf{2 - 8 - 2}$ & $0 \%$ & $30.76 \%$ & $\mathbf{3 0 . 7 6 \%}$ & $30.76 \%$ & $\mathbf{6 1 . 5 2 \%}$ \\
\hline $\mathbf{2 - 1 6 - 2}$ & $0 \%$ & $23.07 \%$ & $\mathbf{2 3 . 0 7 \%}$ & $61.52 \%$ & $\mathbf{8 4 . 5 9 \%}$ \\
\hline $\mathbf{2 - 3 2 - 2}$ & $0 \%$ & $15.38 \%$ & $\mathbf{1 5 . 3 8 \%}$ & $46.14 \%$ & $\mathbf{6 1 . 5 2 \%}$ \\
\hline $\mathbf{2 - 6 4 - 2}$ & $0 \%$ & $7.69 \%$ & $\mathbf{7 . 6 9 \%}$ & $53.83 \%$ & $\mathbf{6 1 . 5 2 \%}$ \\
\hline $\mathbf{2 - 1 2 8 - 2}$ & $0 \%$ & $30.76 \%$ & $\mathbf{3 0 . 7 6 \%}$ & $15.38 \%$ & $\mathbf{4 6 . 1 4 \%}$ \\
\hline
\end{tabular}

Table 5. Results obtained by BLX-0.1 with GMD+STB

\begin{tabular}{|c|c|c|c|c|c|}
\hline BLX 0.1 & BA/BT & BA/ST & TB & ST/NBA & T B/S \\
\hline $\mathbf{2 - 2 - 2}$ & $0 \%$ & $0 \%$ & $\mathbf{0 \%}$ & $23.07 \%$ & $\mathbf{2 3 . 0 7 \%}$ \\
\hline $\mathbf{2 - 4 - 2}$ & $7.69 \%$ & $0 \%$ & $\mathbf{7 . 6 9 \%}$ & $15.38 \%$ & $\mathbf{2 3 . 0 7 \%}$ \\
\hline $\mathbf{2 - 6 - 2}$ & $0 \%$ & $30.76 \%$ & $\mathbf{3 0 . 7 6 \%}$ & $38.45 \%$ & $\mathbf{6 9 . 2 1 \%}$ \\
\hline $\mathbf{2 - 8 - 2}$ & $23.07 \%$ & $38.45 \%$ & $\mathbf{6 1 . 5 2 \%}$ & $30.76 \%$ & $\mathbf{9 2 . 2 8 \%}$ \\
\hline $\mathbf{2 - 1 6 - 2}$ & $0 \%$ & $7.69 \%$ & $\mathbf{7 . 6 9 \%}$ & $7.69 \%$ & $\mathbf{1 5 . 3 8 \%}$ \\
\hline $\mathbf{2 - 3 2 - 2}$ & $0 \%$ & $0 \%$ & $\mathbf{0 \%}$ & $15.38 \%$ & $\mathbf{1 5 . 3 8 \%}$ \\
\hline $\mathbf{2 - 6 4 - 2}$ & $0 \%$ & $0 \%$ & $\mathbf{0 \%}$ & $7.69 \%$ & $\mathbf{7 . 6 9 \%}$ \\
\hline $\mathbf{2 - 1 2 8 - 2}$ & $0 \%$ & $0 \%$ & $\mathbf{0 \%}$ & $23.07 \%$ & $\mathbf{2 3 . 0 7 \%}$ \\
\hline
\end{tabular}

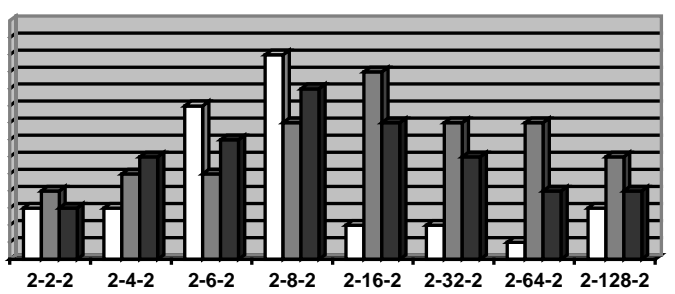

Fig. 4. Results of BLX- $\alpha$ with GMD+STB for $\alpha=0.1,0.3$, and 0.5 
According to the Tables 3-5 and the Figure 4, we may see that BLX- $\alpha$ with GMD+STB achieves the best results when the number of descendants is eight, for $\alpha=0.1$ and $\alpha=0.5$, and sixteen for $\alpha=0.3$. The results improve gradually as the descendants number increases until 8 or 16 descendants are reached. From this number onwards, the results are progressively worse. It is clear that a high number of descendants produces a worse behavior. A higher number of descendants produces a higher selective pressure, which induces a loss of diversity.

Since each parameter obtains the best results with a different number of descendants, we have compared BLX-0.1 and BLX-0.5 with eight descendants, and BLX-0.3 with sixteen descendants, with the aim of deciding which of them is the best. Table 6 shows this comparison.

Table 6. Comparison between different parameters and descendants number

\begin{tabular}{|c|c|c|c|c|c|}
\hline Crossover & BA/BT & BA/ST & TB & ST/NBA & T B/S \\
\hline BLX-0.1 (2-8-2) & $0 \%$ & $46.14 \%$ & $\mathbf{4 6 . 1 4 \%}$ & $15.38 \%$ & $\mathbf{6 1 . 5 2 \%}$ \\
\hline BLX-0.3 (2-16-2) & $38.45 \%$ & $23.07 \%$ & $\mathbf{6 1 . 5 2 \%}$ & $15.38 \%$ & $\mathbf{7 6 . 9 \%}$ \\
\hline BLX-0.5 (2-8-2) & $0 \%$ & $46.14 \%$ & $\mathbf{4 6 . 1 4 \%}$ & $15.38 \%$ & $\mathbf{6 1 . 5 2 \%}$ \\
\hline
\end{tabular}

We observe that the best results are obtained when the $\alpha$ parameter is 0.3 and the operator generates sixteen descendants.

\section{Study of the Selection of Descendents: STB versus Selection of All Descendents}

The objective of this section is to study the importance of the STB strategy as mechanism for selecting the descendents that are introduced in the population. In order to do this, we attempt to determine whether the generation of multiple descendents may work adequately along with a different strategy. In particular, we investigate an approach where all the descendants generated for every pair of parents are included in the new population. The pairs of parents that do not generate descendants, according to the crossover probability, are incorporated in the population, as well. The process finishes when the population reaches 61 elements. We have carried out the experiments with BLX- $\alpha$, assuming $\alpha=0.5$, which is the most standard value ([4]). Table 7 shows the results achieved using different values for the number of descendents.

The alternative strategy does not obtain suitable results when multiple descendants are generated. This does not occur with the STB strategy (Section 3), which has arisen as a powerful technique for working together with the generation of multiple descendents (Section 4). 
Table 7. Results obtained by the BLX-0.5 with the new strategy investigated

\begin{tabular}{|c|c|c|c|c|c|}
\hline Num. Desc. & BA/BT & BA/ST & TB & ST/NBA & T B/S \\
\hline $\mathbf{2}$ & $92.28 \%$ & $7.69 \%$ & $\mathbf{1 0 0 \%}$ & $0 \%$ & $\mathbf{1 0 0 \%}$ \\
\hline $\mathbf{4}$ & $0 \%$ & $0 \%$ & $\mathbf{0 \%}$ & $0 \%$ & $\mathbf{0 \%}$ \\
\hline $\mathbf{6}$ & $0 \%$ & $0 \%$ & $\mathbf{0 \%}$ & $0 \%$ & $\mathbf{0 \%}$ \\
\hline $\mathbf{8}$ & $0 \%$ & $0 \%$ & $\mathbf{0 \%}$ & $0 \%$ & $\mathbf{0 \%}$ \\
\hline $\mathbf{1 6}$ & $0 \%$ & $0 \%$ & $\mathbf{0 \%}$ & $0 \%$ & $\mathbf{0 \%}$ \\
\hline $\mathbf{3 2}$ & $0 \%$ & $0 \%$ & $\mathbf{0 \%}$ & $0 \%$ & $\mathbf{0 \%}$ \\
\hline $\mathbf{6 4}$ & $0 \%$ & $0 \%$ & $\mathbf{0 \%}$ & $7.69 \%$ & $\mathbf{7 . 6 9 \%}$ \\
\hline
\end{tabular}

\section{Study with Different Numbers of Evaluations}

In this section, we analyze whether the results obtained by GMD+STB may be influenced by the number of evaluations considered. We have carried out additional experiments with different values for the number of evaluations $(50000,150000$, and 200000).

Figure 5 shows the percentages in which BLX-0.5 with GMD+STB has obtained the best results with 50000, 100000, 150000, and 200000 evaluations (according to the number of descendants).
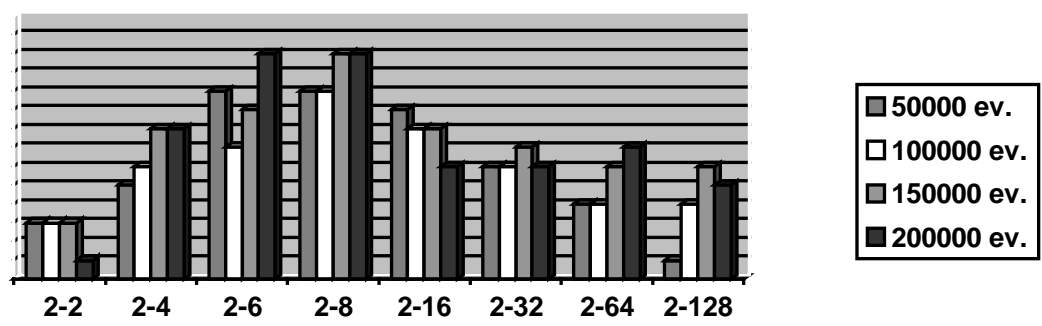

Fig. 5. BLX-0.5 with GMD+STB for 50000, 100000, 150000, and 200000 evaluations. We may remark that the generation of multiples descendants achieves good results independently of the number of evaluations. This property is limited by a particular offspring number. If too many descendants are generated, the selective pressure is very high after the selection of the two best ones. In this way, a disproportionate balance is produced between the exploration of the mechanism of generation of children and the exploitation associated with the selection of the two best ones. 


\section{Conclusions}

We may conclude that the proposal of multiple descendants achieves good results. With the GMD+STB model, the 2-8-2 and 2-16-2 mechanisms for BLX- $\alpha$ obtains better results than the operators with a higher number of descendants, reaching a suitable balance between diversity and selective pressure.

Furthermore, we have conducted experiments with GMD+STB considering others crossover operators for RCGAs. For crossover operators that show exploitation properties, such as the arithmetical crossover operator, the increment of the number of descendants produce an increment of the selective pressure, and no good results are obtained. In contrast, the crossover operators that have exploration properties achieved good results.

\section{References}

1. J.E. Baker: Adaptative Selection Methods for Genetic Algorithms. Proc. Of the First Int. Conf. On Genetic Algorithms and their Applications. J.J. Grefenstette (Ed.) (L. Erlbraum Associates, Hillsdale, MA, 1987), 14-21.

2. J.E. Baker: Reducing bias and inefficiency in the selection algorithm. Proc. Of the Second Int. Conf. On Genetic Algorithms and their Applications, J.J. Grefenstette, Ed., Hillsdale, NJ: Lawrence Erlbaum, 1987, 14-21.

3. K. Deb, D. Joshi, A. Anand: Real Coded Evolutionary Algorithms with Parent-Centric Recombination. KanGAL Report Number 20001003. Indian Institute of Technology, Kanpur, 2001.

4. L.J. Eshelman, J.D. Schaffer: Real-Coded Genetic Algorithms and Interval-Schemata. Foundations of Genetic Algortihms 2, L.D. Whitley (Ed.) (Morgan Kaufmann Publishers, San Mateo, 1993), 187-202.

5. S. Esquivel, R. Gallard, Z. Michalewicz: MCPC: Another Approach to Crossover in Genetic Algorithms. Actas del Primer Congreso Argentino de Ciencias de la Computación, 1995, 141-150.

6. S. Esquivel, A. Leiva, R. Gallard: Multiple Crossover per Couple in Genetic Algorithms. Proc. of the $4^{\text {th }}$ IEEE Int. Conf. on Evolutionary Computation (ICEC'97), Indianapolis, USA, 1997, 103-106.

7. S. Esquivel, S. Ferrero, R. Gallard, C. Salto, H. Alfonso, M. Schütz: Enhanced Evolutionary Algorithms for Single and Multiobjective Optimization in the Job Shop Scheduling Problem. Journal of Knowledge Based Systems 15:1-2 (2002) 12-25.

8. F. Herrera, M. Lozano, J.L. Verdegay: Dynamic and Heuristic Fuzzy Connectives-Based Crossover Operators for Controlling the Diversity and Convergence of Real Coded Genetic Algorithms. International Journal of Intelligent Systems 11 (1996) 1013-1041.

9. F. Herrera, M. Lozano, J.L. Verdegay: Fuzzy Connectives Based Crossover Operators to Model Genetic Algorithms Population Diversity. Fuzzy Sets and Systems 92:1 (1997) 21-30.

10. F. Herrera, M. Lozano, J.L. Verdegay: Tackling Real-Coded Genetic Algorithms: Operators and Tools for the Behaviour Analysis. Artificial Intelligence Review 12 (1998) 265319. 
11. F. Herrera, M. Lozano: Gradual Distributed Real-Coded Genetic Algorithms. IEEE Transactions on Evolutionary Computation 4:1 (2000) 43-63.

12. Z. Michalewicz: Genetic Algorithms + Data Structures = Evolution Programs. SpringerVerlag, New York (1992).

13. R.C. Reynolds, C. Chung: Knowledge-Based Self-Adaptation in Evolutionary Programming Using Cultural Algorithms. Proc. 1997 IEEE International Conference on Evolutionary Computation, IEEE Press, 1997, 71-76.

14. H. Satoh, M. Yamamura, S. Kobayashi: Minimal Generation Gap Model for GAs Considering Both Exploration and Exploitation. Proc. Of IIZUKA: Methodologies for the Conception, Design and Application of Intelligent Systems, 1996, 494-497.

15. A. Wright: Genetic Algorithms for Real Parameter Optimization. Foundations of Genetic Algorithms I, G.J.E. Rawlin (Ed.) (Morgan Kaufmann, San Mateo,1991), 205-218. 